\title{
Erratum to: Self-adjustable hyper-graphs for video pose estimation based on spatial-temporal subspace construction
}

\author{
Jizhou MA ${ }^{1}$, Shuai $\mathrm{LI}^{1 *}$, Hong $\mathrm{QIN}^{2}$, Aimin $\mathrm{HAO}^{1}$ \& Qinping $\mathrm{ZHAO}^{1}$ \\ ${ }^{1}$ State Key Laboratory of Virtual Reality Technology and Systems, Beihang University, Beijing 100191, China; \\ ${ }^{2}$ Department of Computer Science, Stony Brook University, New York 11794, USA \\ Received 20 July 2021/Accepted 30 July 2021/Published online 10 August 2021

Citation Ma J Z, Li S, Qin H, et al. Erratum to: Self-adjustable hyper-graphs for video pose
estimation based on spatial-temporal subspace construction. Sci China Inf Sci, 2022, 65(3): 139901,
https://doi.org/10.1007/s11432-021-3298-5

In the original publication [1] of this paper, the third author Hong QIN is associated only with the Department of Computer Science, Stony Brook University, New York, 11794, USA.

\section{References}

1 Ma J Z, Li S, Qin H, et al. Self-adjustable hyper-graphs for video pose estimation based on spatial-temporal subspace construction. Sci China Inf Sci, 2022, 65: 139101

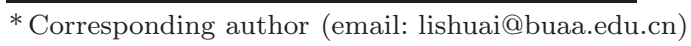

